ГНЕДЫХ Д. С.

ПРОБЛЕМА ОЦЕНКИ ЭКВИВАЛЕНТНОСТИ КОМПЬЮТЕРНЫХ И ТРАДИЦИОННЫХ ВЕРСИЙ...

Российский психологИчЕский жУРнАл, 2020, Т. 17, № 1, 44-59. doi: 10.21702/rpj.2020.1.4

ОБЩАЯ ПСИХОЛОГИЯ, ПСИхОЛОГИЯ лИЧнОстИ, ИСТОРИя ПСИхОЛОГИИ

УдК 159.9.07 doi: $10.21702 /$ rpj.2020.1.4

Систематический обзор

\title{
Проблема оценки эквивалентности компьютерных и традиционных версий психодиагностических методик
}

\author{
Дарья С. Гнедых \\ Санкт-Петербургский государственный университет, г. Санкт-Петербург, Российская Федерация \\ E-mail: d.gnedyh@spbu.ru \\ ORCID ID: https://orcid.org/0000-0003-4955-4779
}

\begin{abstract}
Аннотация
Введение. В статье рассматриваются теоретико-метолологические основания АоказательСтва ЭКвивалентности КомПьютерной и траАиционной фформ психоАИагностических МетоАИк. ПреАставлен анализ исслеАОваний психометрических показателей компьютерных версий

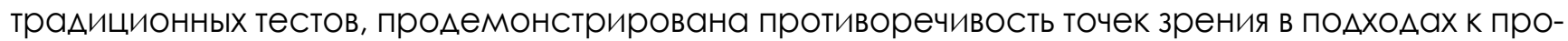
верке эквивалентности Авух фрорм метолик. Научная новизна заключается в попытке структурирования основных проблем, возникающих в процессе установления эквивалентности компьютерной и бумажной версий методик, а также поиске путей и способов их преодоления. Поднимается вопрос о минимальном, но Аостаточном наборе математико-статистических

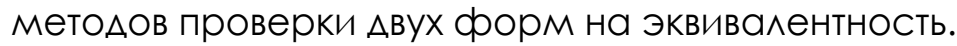

Теоретическое обоснование. Приведены основные проблемы, связанные с оценкой эквива^ентности компьютерной и традиционной фрорм теста: уровень общекультурной и инфрормационной компетентности респондентов, тревожность, социальное окружение, мотивация к проведению тестирования, С^ожность в организации одинаковых условий при заполнении компьютерных и «бланковых» фрорм метолик (^. Н. Бабанин, Ү. P. Chua, M. Russell, P. Květon и Ар.). В качестве методов оценки эквивалентности Авух фрорм теста исслеАователи чаще всего выбирают: сравнение основных статистических показателей (среАних значений, Аисперсий и Ар.), проверку конструктной валидности и надежности компьютерной версии психоАИагностической метоАики.

Результаты и их обсужАение. В результате анализа метоАологии исслеАований, посвященных оценке эквивалентности компьютерных и традиционных фрорм тестов, было выявлено разнообразие полхоАов: к применению математико-статистических метолов Аля проверки

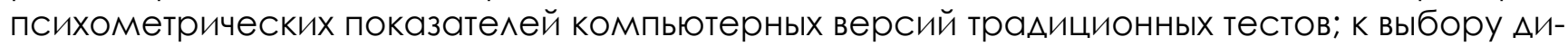
зайна исслеАования; по отношению к учету специфики ситуации компьютерной психодиагностики. Аелается вывоА О необходимости фоормулировки конкретных и структурированных требований к процеАуре проверки эквивалентности компьютерной и траАиционной фрорм психодиагностических метолик. ПреАложены рекоменАации касательно основных разделов, которые Аолжны войти в Аанные требования: организация эмпирического исследования, математико-статистические метолы, контроль фракторов, специфиичных Аля компьютерной психодиагностики и способных оказать влияние на результаты оценки эквивалентности.
\end{abstract}

\section{КАючевые слова}

психодиагностические методики, психометрические показатели, эквивалентность тестов, 
ГНЕДЫХ Д. С.

ПРОБЛЕМА ОЦЕНКИ ЭКВИВАЛЕНТНОСТИ КОМПЬЮТЕРНЫХ И ТРАДИЦИОННЫХ ВЕРСИЙ...

РосСИЙСКИй пСИХологИЧЕСКИй ЖУРнАл, 2020, Т. 17, № 1, 44-59. doi: 10.21702/rpj.2020.1.4

ОБЩАЯ ПСИХОЛОГИЯ, ПСИХОЛОГИЯ ЛИЧНОСТИ, ИСТОРИЯ ПСИХОЛОГИИ

математико-статистические методы, компьютеризация тестов, компьютерное тестирование, компьютерная психодиагностика, методология исслеАования, валиАность, наАежность теста

\section{Основные положения}

จ оАной из метоАологических проблем проверки психометрической эКвивалентности компьютерной и бумажной фрорм методик явАяется отсутствие стандартизации Аанного процесса; > А^я унификации процесса оценки эквивалентности компьютерной и траАиционной фрорм психодиагностических метолик необходимо разработать еАиные требования к Аизайну исслеАования, а также к метоАам математико-статистической обработки Аанных;

$\checkmark$ Аля учета специорических фракторов ситуации компьютерного тестирования исследовате^ям рекоменАуется пользоваться картами наблюАения и^и анкетами на выявление уровня компьютерной грамотности респонАентов, их мотивации и установок по отношению к компьютерной психодиагностике.

\section{Для цитирования}

Гнедых, Д. С. (2020). Проблема оценки эквивалентности компьютерных и традиционных версий психодиагностических методик. Российский психологический журнал, 17(1), 44-59. doi: 10.21702/rpj.2020.1.4

Дата получения рукописи: 28.01.2020 Дата окончания рецензирования: 29.02.2020 Дата принятия к публикации: 04.03.2020

\section{Введение}

С ростом темпа жизни, увеличением объема информации и технических возможностей возникает необходимость в поиске способов экономии времени при проведении психологических исследований. Как следствие, происходит перевод «бланковых» (бумажных, или традиционных) психодиагностических методик в компьютерные версии, что позволяет собирать информацию дистанционно, а также автоматизировать процесс обработки данных. Для этих целей используются различные сервисы-Google-формы, конструкторы онлайн-опросов или специализированные программы. В большинстве случаев перевод (из бланка в компьютер) сводится к простому действию - текст опросника (или иного рода стимульный материал) копируется в электронный формат и рассылается всем желающим для заполнения. При этом с интерпретацией результатов поступают схожим образом - нормы «бланковых» методик применяются к их компьютерным «аналогам».

В научном сообществе уже давно поднимается вопрос, насколько эквивалентными являются компьютерная и традиционная формы психодиагностической методики. Mazzeo, Druesne, Raffeld, Checketts, \& Muhlstein (1992) придерживаются мнения о том, что необходимо проводить специальные исследования сопоставимости показателей данных форм. При этом за всю историю вопроса наблюдаются противоречия во взглядах на то, какие именно психометрические показатели теста следует подвергать проверке. Существует мнение, что если доказать эквивалентность двух форм, то и валидности их будут эквивалентны (George, Lankford, \& Wilson, 1992; Ford, Vitelli, \& Stuckless, 1996). Однако L. M. Honaker прямо указывает на то, что показатели валидности, определенные для бумажной формы, не могут автоматически 
ГНЕДЫХ Д. С.

ПРОБЛЕМА ОЦЕНКИ ЭКВИВАЛЕНТНОСТИ КОМПЬЮТЕРНЫХ И ТРАДИЦИОННЫХ ВЕРСИЙ...

Российский психологИчЕский жУРнАл, 2020, Т. 17, № 1, 44-59. doi: 10.21702/rpj.2020.1.4

ОБЩАЯ ПСИХОЛОГИЯ, ПСИХОЛОГИЯ ЛИЧНОСТИ, ИСТОРИЯ ПСИХОЛОГИИ

переноситься на компьютерную (Honaker, 1988), и в таком случае следует проводить дополнительные исследования валидности (Russell, Goldberg, \& O'connor, 2003). С ним солидарны Анастази и Урбина (2009, с. 93): «...надежность и валидность теста могут варьировать в зависимости от формы предъявления». Батурин и Мельникова (2011a, 20116) говорят о том, что процедура создания компьютерной версии методики - не простое копирование стимульного материала из одной формы в другую, а модификация методики, что подразумевает процесс ее переработки. Такую точку зрения поддерживает ряд других ученых: «компьютерная версия методики - это вполне самостоятельная методика, использование которой возможно только после установления групповых норм и других психометрических характеристик» (Вассерман, Иовлев и Червинская, 2010, с. 23). Более того, некоторые исследователи предполагают, что совпадение баллов, набранных по бумажной и компьютерной формам теста, не говорит о том, что обе формы измеряют один и тот же психологический конструкт (что было показано на примере личностных методик) (Meade, Michels, \& Lautenschlager, 2004). Несмотря на то, что на данную проблему обратили внимание еще в конце XX в., не все исследователи, как будет показано ниже, проводят полную проверку психометрических параметров компьютерной формы традиционной методики, математико-статистические методы анализа их эквивалентности довольно разнообразны, а выбор данных методов часто не обосновывается авторами.

Наличие разнообразия мнений относительно набора психометрических показателей компьютеризированного теста, требующих проверки, отчасти объясняется отсутствием четких универсальных инструкций по процедуре установления эквивалентности компьютерной и бумажной форм психодиагностических методик. Существуют рекомендации по созданию психодиагностического инструментария в целом, которые позволяют структурировать накопленную в этой области информацию и облегчить работу психологам и другим специалистам, встающим на этот путь (хорошим примером, на наш взгляд, является серия статей Батурина и Мельниковой (2009-2011); American Educational Research Association, American Psychological Association, \& National Council on Measurement in Education (1999) (AERA, APA, NCME)). Проверка эквивалентности компьютерной версии теста его бумажному аналогу - это отдельная специфическая область психодиагностики, которая также требует определенности и стандартов.

Очевидно, что компьютеризация бланковых методик в будущем будет принимать всё большие масштабы. Одновременно нарастает обеспокоенность научного сообщества по поводу валидности компьютерных версий «бланковых» тестов. Таким образом, возникает необходимость стандартизации и контроля процесса перевода бумажных методик в компьютерные формы.

В статье рассматривается опыт проверки психометрических показателей компьютерных форм традиционных психодиагностических методик, поднимается вопрос о минимальном наборе математико-статистических методов проверки двух форм на эквивалентность, а также о необходимости формализации процедуры фиксации специфических условий компьютерного тестирования (непосредственно при проведении исследования на эквивалентность) и их последующего учета при интерпретации результатов.

\section{Теоретическое обоснование}

Проблема влияния различных факторов на процесс и процедуру заполнения компьютерной формы «бланковой» методики активно обсуждается в научном сообществе. Бабанин (2010), указывая на факторы, которые могут повлиять на эквивалентность двух форм методик (наличие компьютерного опыта, тревожность, социальное окружение и др.), приходит к выводу, 
ГНЕДЫХ Д. С.

ПРОБЛЕМА ОЦЕНКИ ЭКВИВАЛЕНТНОСТИ КОМПЬЮТЕРНЫХ И ТРАДИЦИОННЫХ ВЕРСИЙ...

РосСИЙСКИй пСИХологИЧЕСКИй ЖУРнАл, 2020, Т. 17, № 1, 44-59. doi: 10.21702/rpj.2020.1.4

ОБЩАЯ ПСИХОЛОГИЯ, ПСИХОЛОГИЯ ЛИЧНОСТИ, ИСТОРИЯ ПСИХОЛОГИИ

что основным из них является уровень общекультурной и информационной компетентности (уровень освоения информационных технологий). Но учет компьютерной грамотности респондентов всё же не снимает проблемы различия в данных при проверке эквивалентности двух форм методик (Russell et al., 2003). При переносе «бумажных» тестов в компьютерный формат необходимо максимально приблизить условия заполнения методики к таковым для бумажной версии (соблюдать ограничения во времени предъявления стимульного материала, если это требуется в оригинале методики, учесть возможность исправления ответов, строгого/нестрогого порядка выполнения заданий и т. п.). Это один из способов, который может повысить вероятность эквивалентности данных форм.

Следующий момент, который требует внимания,- это выбор математико-статистических методов анализа данных. Какие действия для проверки психометрической эквивалентности компьютерной и бумажной форм методики можно считать необходимыми и достаточными?

За рубежом в 1986 г. вышло руководство по оценке тестов (АРА, 1986), где перечислены основные статистические методы и показатели, позволяющие определить психометрическую эквивалентность компьютерной и традиционной форм теста: описательная статистика (средние значения, дисперсии, распределения и ранговые порядки оценок), конструктная валидность, надежность. Тем не менее, в исследованиях на проверку эквивалентности авторами не всегда соблюдаются все эти пункты, а в частных случаях проводятся дополнительные виды анализа (например, Bartram, 1994; van de Looij-Jansen, Goldschmeding, \& Jan de Wilde, 2006; Květon, Jelínek, Vobořil, \& Klimusová, 2007; Chua, 2012). Чем же всё-таки обусловлен выбор методов проверки психометрических характеристик компьютерных версий традиционных психодиагностических методик? Каков минимальный перечень методов, которые являются достаточными для доказательства эквивалентности компьютерной формы бумажной форме, или ее самостоятельности как инструментария?

В таблице 1 представлен обзор исследований эквивалентности компьютерных и бумажных форм тестов за последние 20 лет. В качестве критериев анализа выступали: дизайн исследования; применение тех или иных статистических методов для обработки результатов; обоснование их выбора или цели их применения; учет специфики процедуры компьютерного тестирования (проводилось ли наблюдение за респондентами во время выполнения тестов на компьютере, заполнялись ли дополнительные анкеты на выявление отношения участников исследования к компьютерному тестированию в случае, если оно проходило через Интернет, и т. п.).

\section{Результаты и их обсуждение}

Как видно из таблицы 1, при сравнении бумажных и компьютерных форм одного и того же теста, исследователи задаются разными вопросами. Одни проверяют только надежность компьютерного теста, по умолчанию принимая факт, что он валиден; другие считают достаточным провести корреляционный анализ и сравнение средних значений, чтобы доказать эквивалентность; третьи предпочитают сразу проверить все психометрические параметры компьютерной формы. Возможно ли, чтобы выбор статистических методов зависел от специфики стимульного материала? Кажется, что если методика состоит из вопросов (утверждений) и вариантов ответов (по типу опросника), то при переносе ее в компьютерную форму серьезных изменений произойти не должно. К такому выводу приходят некоторые исследователи: надежность многошкальных тестов сохраняется при их переносе с бланка в компьютеризированную форму (Ромек и Сатин, 2000). 
ГНЕДЫХ Д. С.

ПРОБЛЕМА ОЦЕНКИ ЭКВИВАЛЕНТНОСТИ КОМПЬЮТЕРНЫХ И ТРАДИЦИОННЫХ ВЕРСИЙ...

Российский психологИчЕский жУРнАл, 2020, Т. 17, № 1, 44-59. doi: 10.21702/rpj.2020.1.4

\begin{tabular}{|c|c|c|c|c|c|c|c|}
\hline 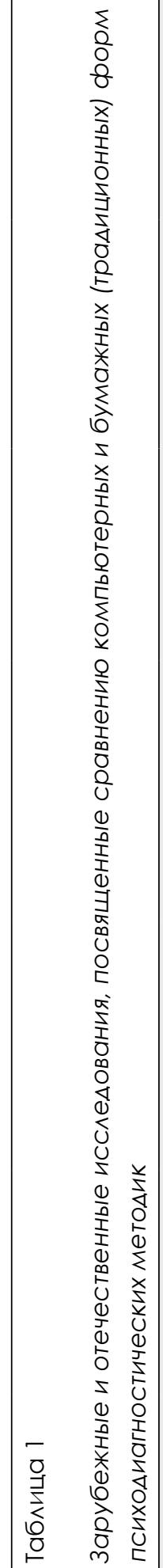 & 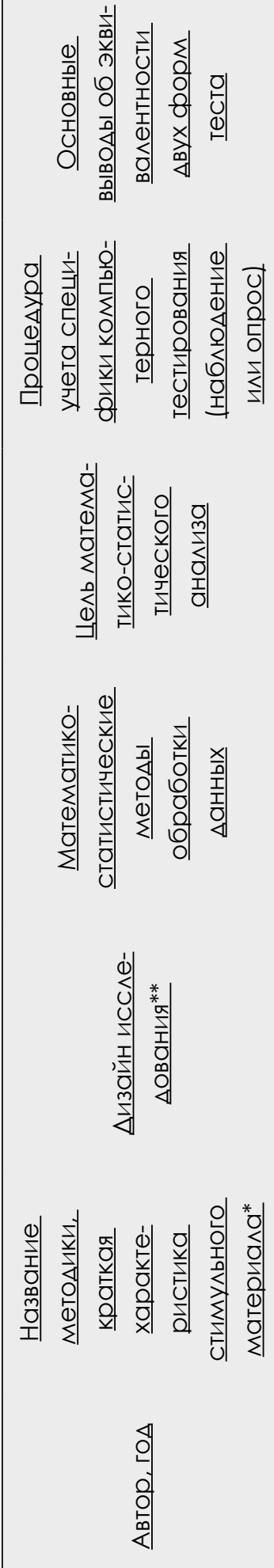 & 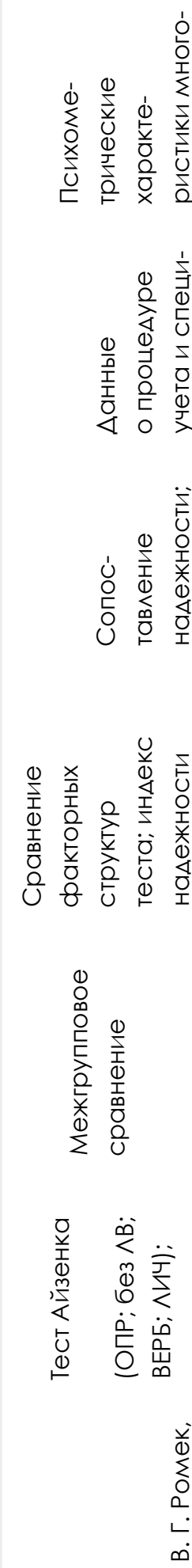 & 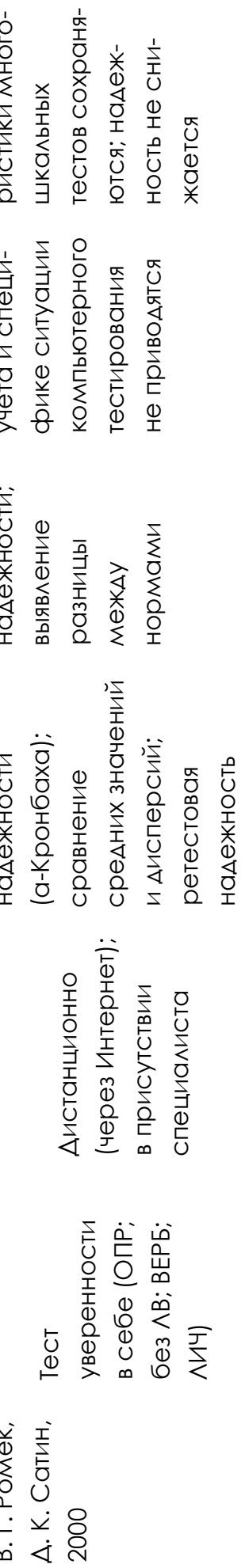 & 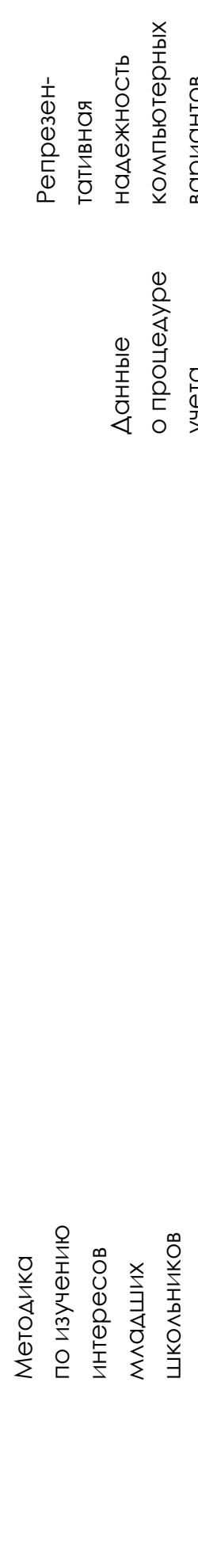 & 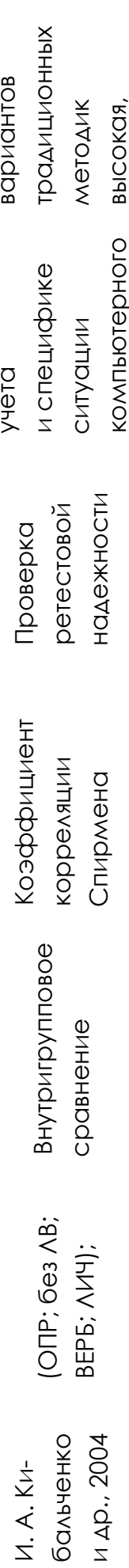 & 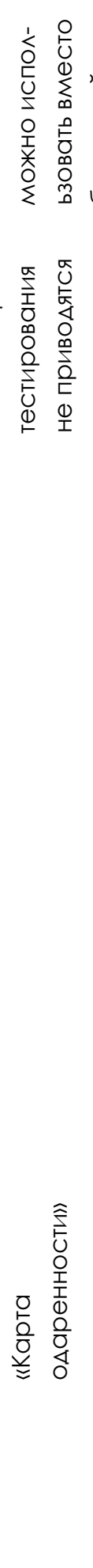 & 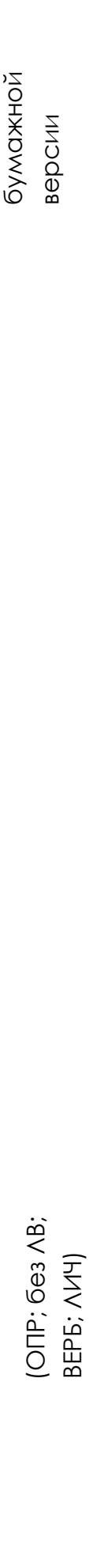 \\
\hline
\end{tabular}


ГНЕДЫХ Д. С.

ПРОБЛЕМА ОЦЕНКИ ЭКВИВАЛЕНТНОСТИ КОМПЬЮТЕРНЫХ И ТРАДИЦИОННЫХ ВЕРСИЙ...

РОССИЙСКИЙ ПСИХОЛОГИЧЕСКИЙ ЖУРНАЛ, 2020, Т. 17, № 1, 44-59. doi: 10.21702/rpj.2020.1.4

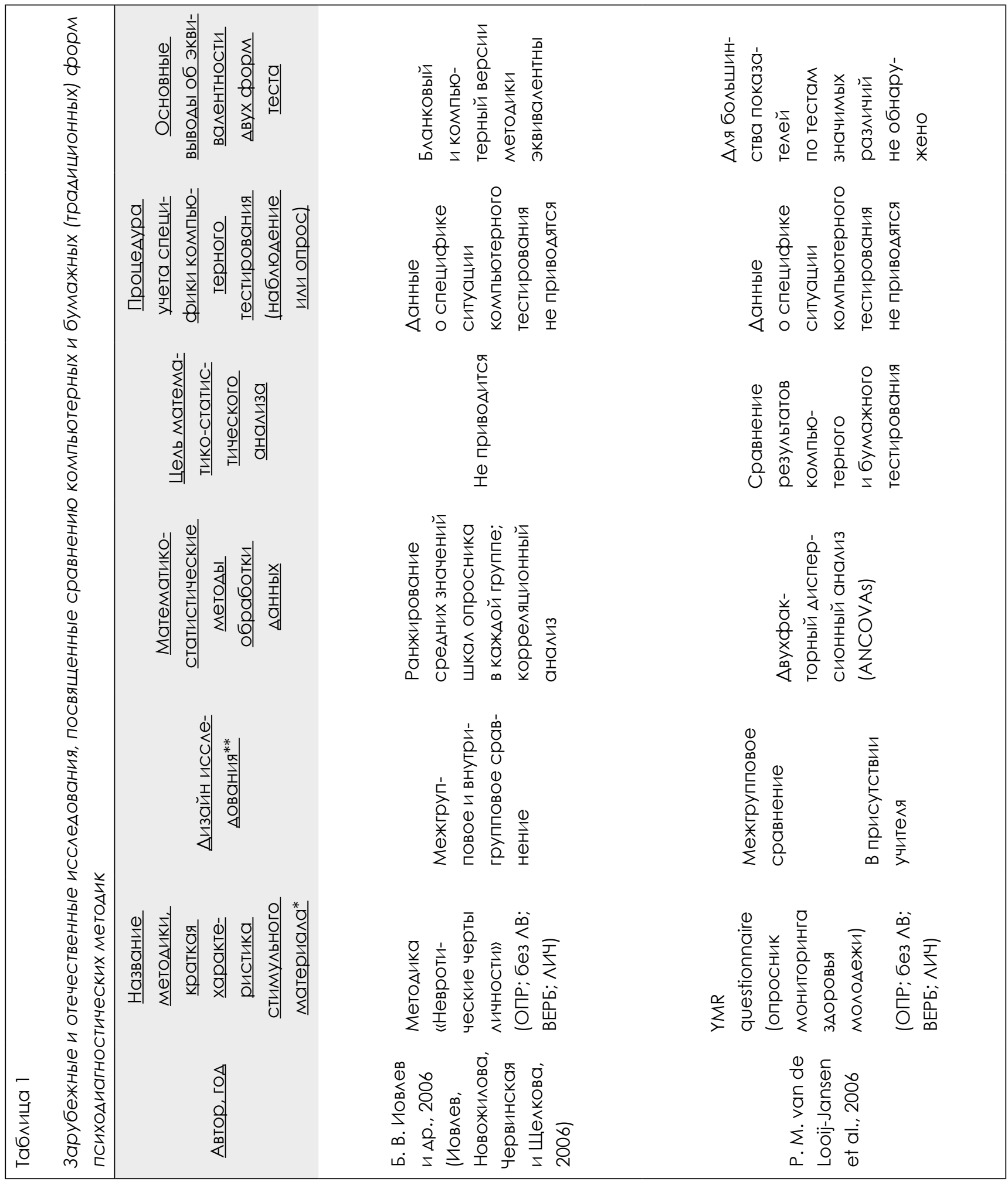


ГНЕДЫХ Д. С.

ПРОБЛЕМА ОЦЕНКИ ЭКВИВАЛЕНТНОСТИ КОМПЬЮТЕРНЫХ И ТРАДИЦИОННЫХ ВЕРСИЙ...

РоссийскиЙ псиХологИЧЕскиЙ ЖХУРнАл, 2020, Т. 17, № 1, 44-59. doi: 10.21702/rpj.2020.1.4

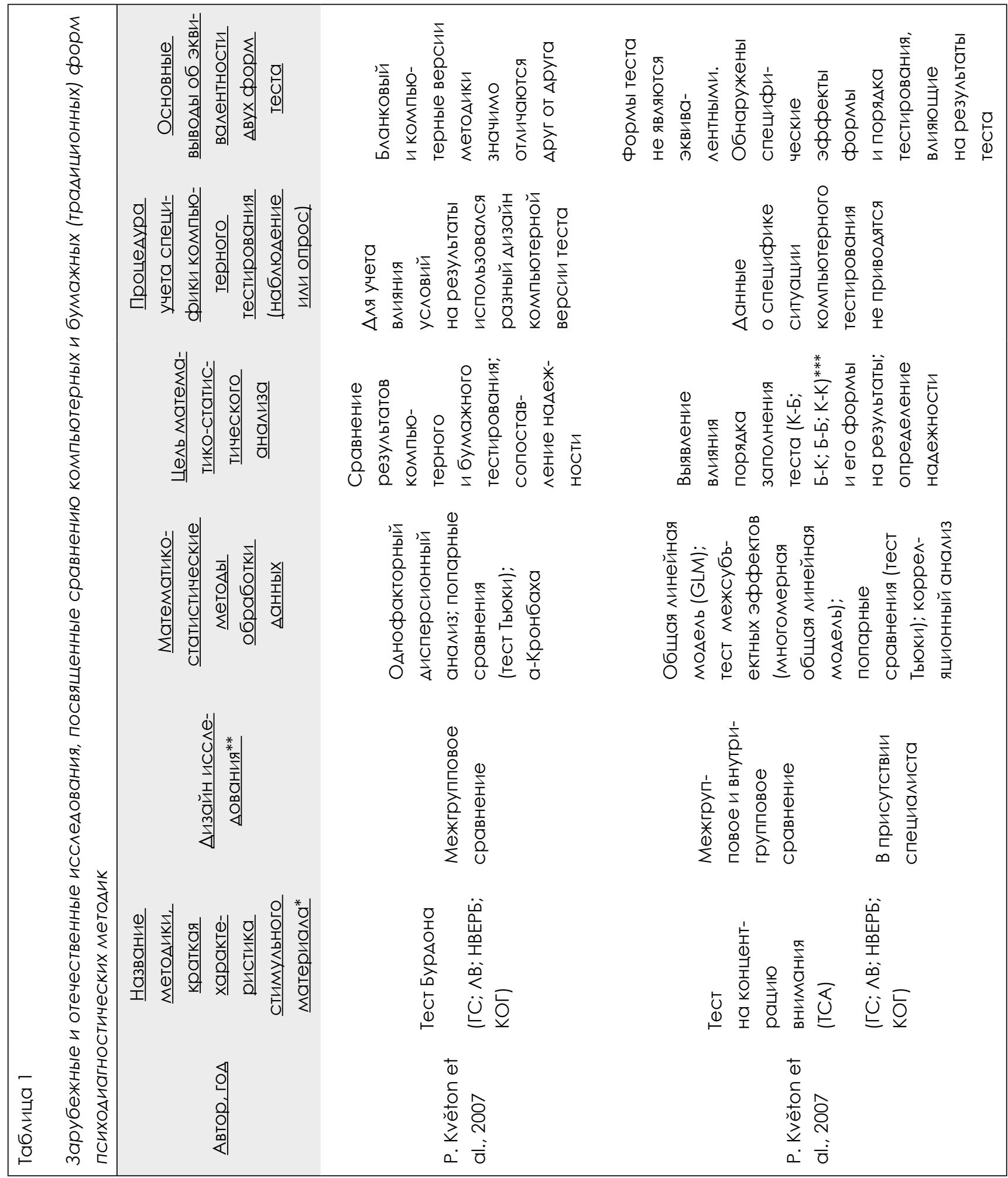


ГНЕДЫХ Д. С.

ПРОБЛЕМА ОЦЕНКИ ЭКВИВАЛЕНТНОСТИ КОМПЬЮТЕРНЫХ И ТРАДИЦИОННЫХ ВЕРСИЙ...

РОССИЙСКИЙ ПСИХОЛОГИЧЕСКИЙ ЖУРНАЛ, 2020, Т. 17, № 1, 44-59. doi: 10.21702/rpj.2020.1.4

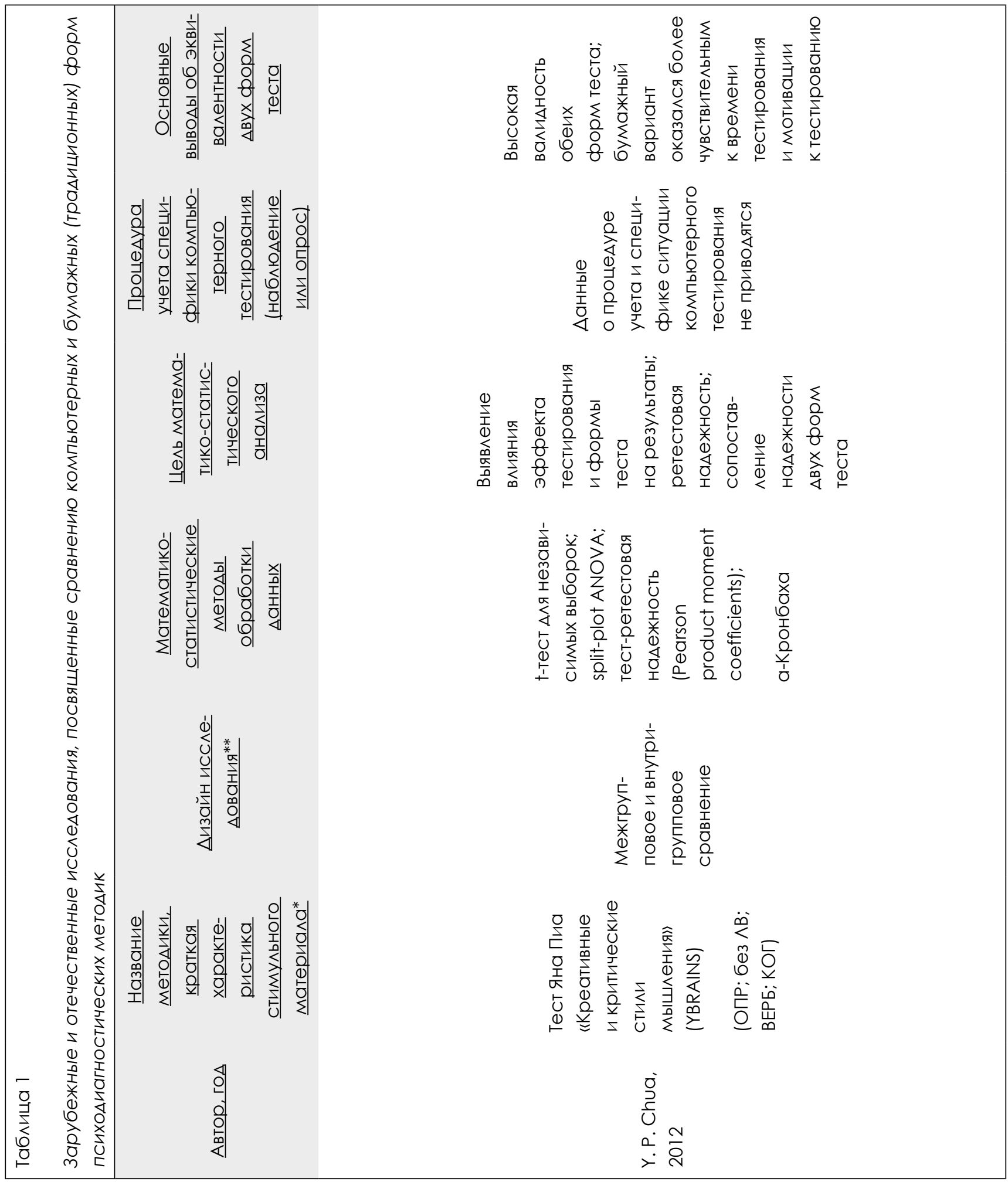


ГНЕДЫХ Д. С.

ПРОБЛЕМА ОЦЕНКИ ЭКВИВАЛЕНТНОСТИ КОМПЬЮТЕРНЫХ И ТРАДИЦИОННЫХ ВЕРСИЙ...

РОссийскИй пСИХОЛОГИЧЕскиЙ ЖУРнАл, 2020, Т. 17, № 1, 44-59. doi: 10.21702/rpj.2020.1.4

\begin{tabular}{|c|c|c|c|}
\hline 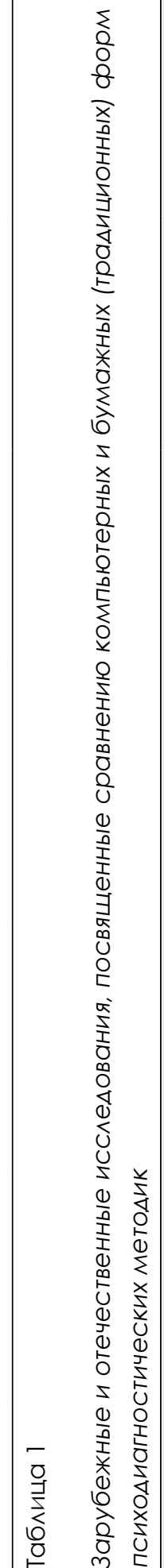 & 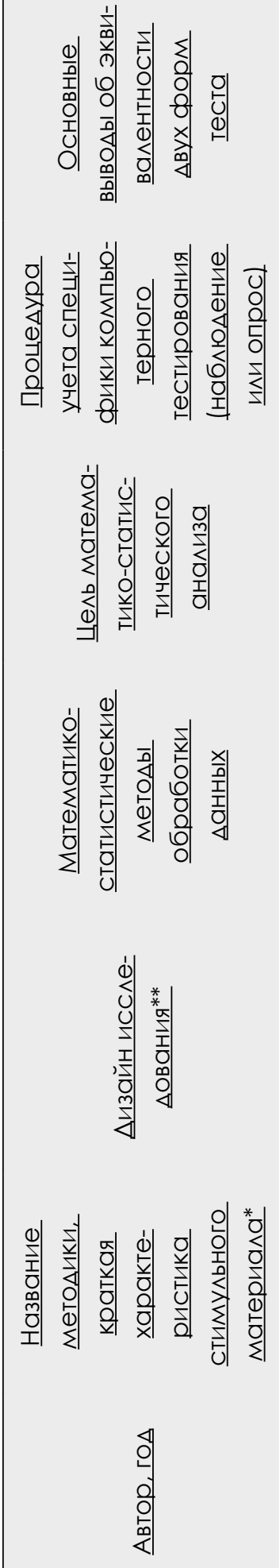 & 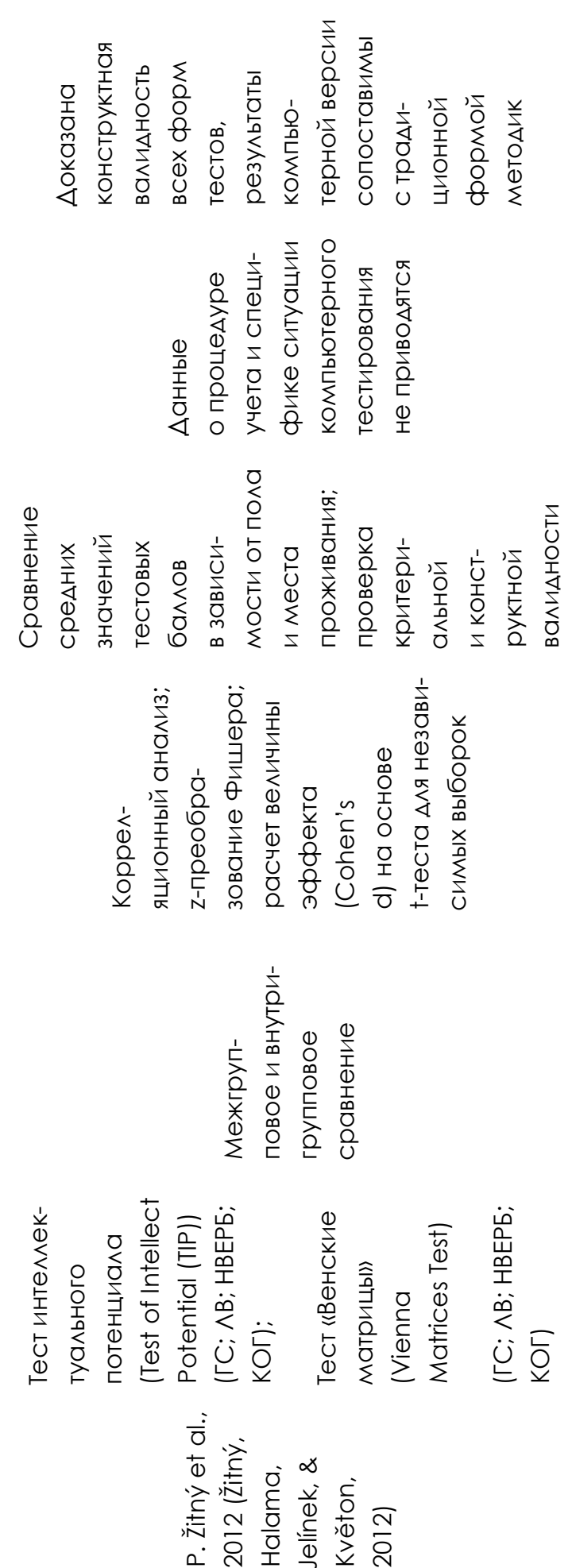 & 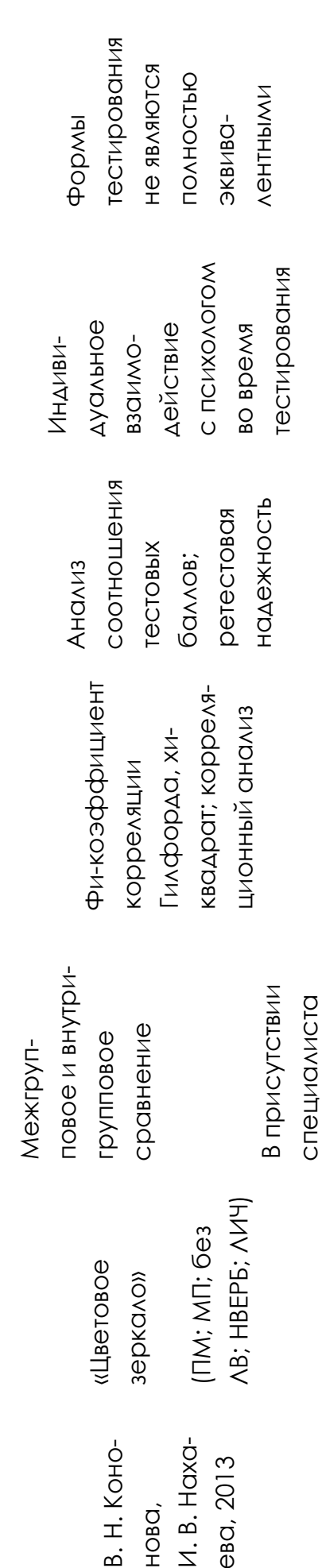 \\
\hline
\end{tabular}


ГНЕДЫХ Д. С.

ПРОБЛЕМА ОЦЕНКИ ЭКВИВАЛЕНТНОСТИ КОМПЬЮТЕРНЫХ И ТРАДИЦИОННЫХ ВЕРСИЙ...

РОССИЙСКИЙ ПСИХОЛОГИЧЕСКИЙ ЖУРНАЛ, 2020, Т. 17, № 1, 44-59. doi: 10.21702/rpj.2020.1.4

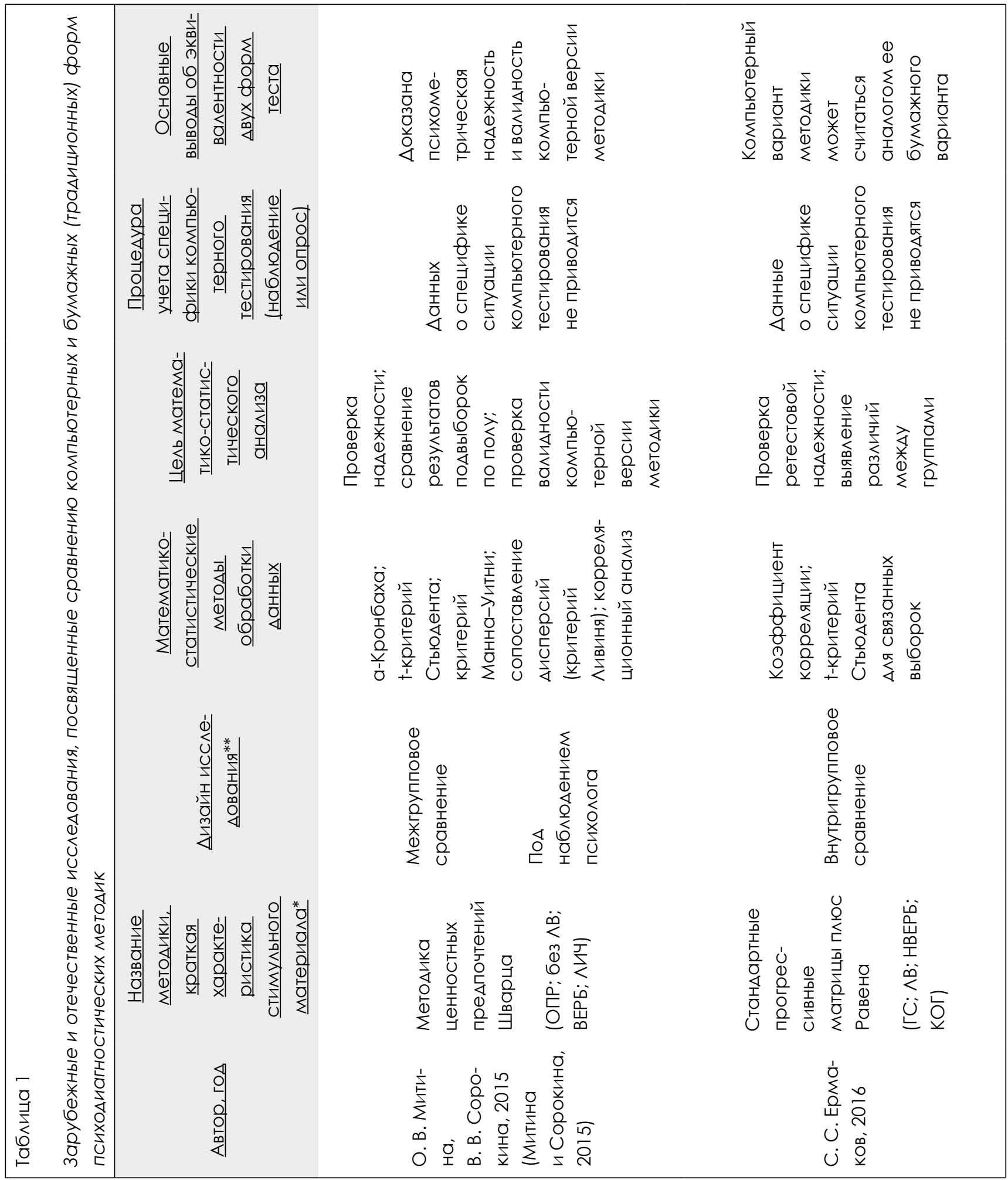


ГНЕДЫХ Д. С.

ПРОБЛЕМА ОЦЕНКИ ЭКВИВАЛЕНТНОСТИ КОМПЬЮТЕРНЫХ И ТРАДИЦИОННЫХ ВЕРСИЙ...

РоссийскиЙ псиХологИЧЕский ЖХУРнАл, 2020, Т. 17, № 1, 44-59. doi: 10.21702/rpj.2020.1.4

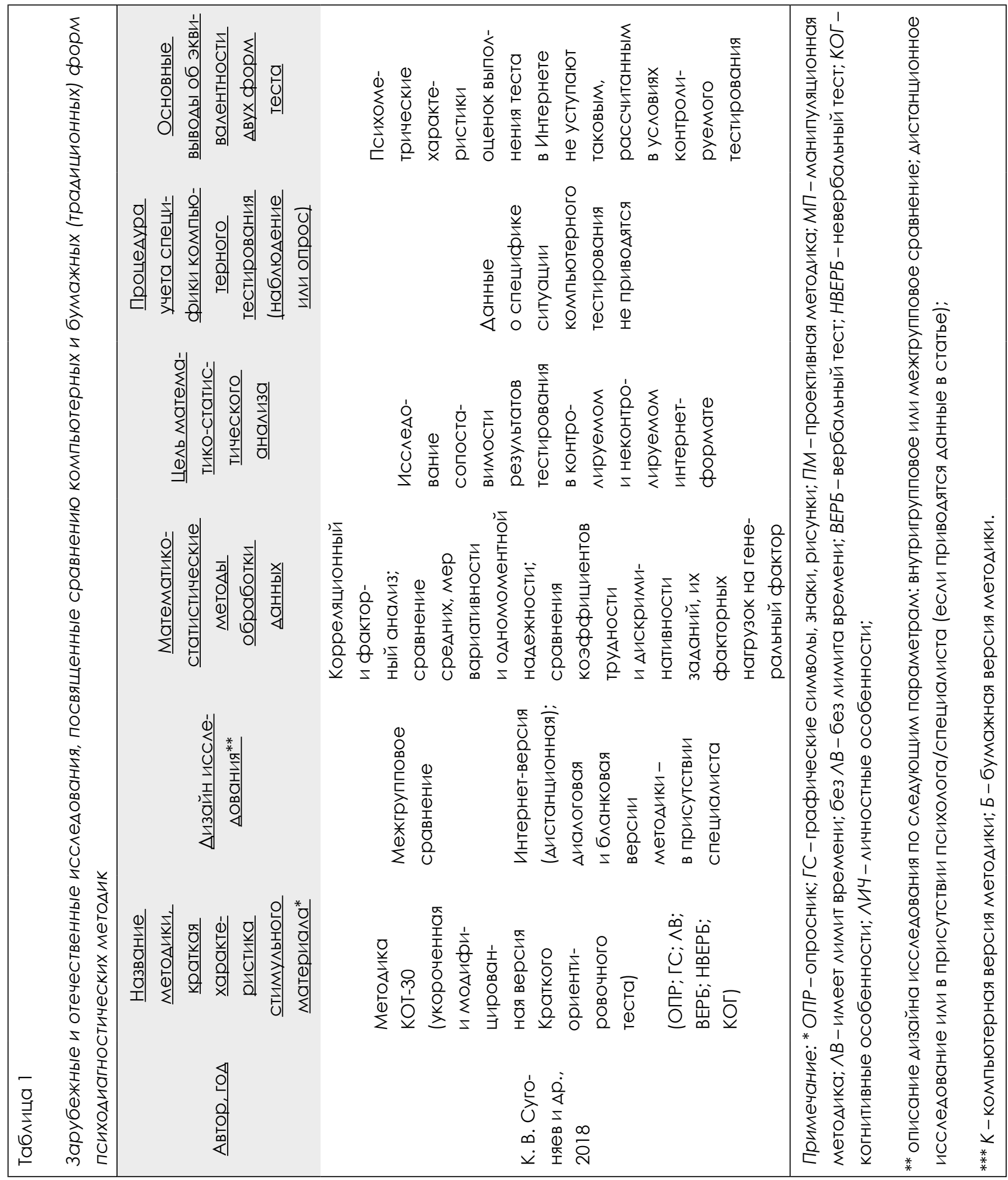


ГНЕДЫХ Д. С.

ПРОБЛЕМА ОЦЕНКИ ЭКВИВАЛЕНТНОСТИ КОМПЬЮТЕРНЫХ И ТРАДИЦИОННЫХ ВЕРСИЙ...

РосСИЙСКИй пСИХологИЧЕСКИй ЖУРнАл, 2020, Т. 17, № 1, 44-59. doi: 10.21702/rpj.2020.1.4

ОБЩАЯ ПСИХОЛОГИЯ, ПСИХОЛОГИЯ ЛИЧНОСТИ, ИСТОРИЯ ПСИХОЛОГИИ

С другой стороны, если стимульный материал представляет собой творческие задания, или методика предполагает работу с картинками, графическими символами, лимит времени и т.д. (как, например, в некоторых когнитивных тестах), то можно предположить, что к проверке психометрических параметров такой компьютерной формы нужно подходить более ответственно. Как показывает анализ исследований, приведенных в таблице, данная логика далеко не всегда лежит в основе выбора статистических методов для обоснования эквивалентности двух форм.

Более того, наблюдается разнообразие подходов к дизайну исследования, призванного проверить эквивалентность двух форм методики (компьютерной и традиционной). Например, в исследованиях Ромека и Сатина (2000), van de Looij-Jansen et al. (2006) используется межгрупповое сравнение (когда одна группа заполняет только бумажную версию методики, а другая - только компьютерную) (см. также Andersson, Kaldo-Sandström, Ström, \& Strömgren, 2003; Vecchione, Alessandri, \& Barbaranelli, 2012), у Ермакова (2016) и Кибальченко, Устинова и Шаповалова (2004) - внутригрупповое (группа заполняет как бумажную, так и компьютерную формы через определенный промежуток времени) (также Hays \& Mccallum, 2005), а где-то - как межгрупповое, так и внутригрупповое сравнение (Květon et al., 2007; Кононова и Нахаева, 2013). Каждый из описанных выше вариантов исследований имеет свои преимущества и недостатки. При этом, на наш взгляд, наличие внутригруппового сравнения в большей степени позволяет контролировать влияние личности респондента на заполнение как бумажной, так и компьютерной версий теста. Такого рода влияние может проявиться в ситуации межгруппового дизайна, когда разница в результатах может быть обусловлена не спецификой формы теста, а различиями в личностных особенностях двух групп респондентов (например, в том случае, когда в одну группу случайно попадает больше тревожных испытуемых, чем в другую).

Наиболее тщательно проверить эквивалентность позволяет также учет дополнительных факторов при процедуре заполнения компьютерной версии методики (Chua, 2012). В связи с этим в таблицу был включен такой критерий анализа содержания статей, как «процедура учета специфики компьютерного тестирования (наблюдение или опрос)». Даже относительно незначительная модификация дизайна методики в компьютерном варианте (например, изменение цветовой схемы) может существенно повлиять на результаты (Květon et al., 2007). Также существует мнение, что когнитивная нагрузка при прохождении компьютерного теста иная, чем при заполнении теста на бумаге, и это приводит к "расслоению выборки", когда худшие показывают результаты еще хуже, а лучшие еще лучше» (Ермаков, 2016, с. 203). К тому же, примирение дизайна пре-тест и пост-тест может привести к проявлению эффекта тестирования (опыт прохождения пре-теста влияет на результаты пост-теста), а исследователь иногда, упуская это из виду, делает вывод о том, что влияние на результаты последнего тестирования было оказано условием прохождения (например, компьютером, если он был вторым по порядку). Во избежание неверных выводов, связанных с влиянием эффекта тестирования, Chua (2012) предлагает использовать экспериментальный план Р. Л. Соломона для четырех рандомизированных групп (подробнее о плане см. Solomon, 1949; Campbell \& Stanley, 1963; и др.). Кроме того, существует еще одна проблема, связанная с проведением компьютерного тестирования, предполагающего удаленный доступ (через Интернет),- это фальсификация результатов (Сугоняев, Радченко и Соколов, 2018). В таком случае рекомендуется перепроверять их в контролируемых условиях (The International Testing Commission, 2006). 
ГНЕДЫХ Д. С.

ПРОБЛЕМА ОЦЕНКИ ЭКВИВАЛЕНТНОСТИ КОМПЬЮТЕРНЫХ И ТРАДИЦИОННЫХ ВЕРСИЙ...

Российский психологИчЕский жУРнАл, 2020, Т. 17, № 1, 44-59. doi: 10.21702/rpj.2020.1.4

ОБЩАЯ ПСИХОЛОГИЯ, ПСИхОЛОГИЯ лИЧнОстИ, ИСТОРИя ПСИхОЛОГИИ

Учет всех этих моментов позволяет более тщательно подойти к продумыванию дизайна исследования - выбору количества групп респондентов, последовательности проведения серий тестирования и т. д. На наш взгляд, также будет полезной организация наблюдения за поведением респондентов во время работы с компьютерной версией теста - это поможет исключить из анализа результаты респондентов, демонстрирующих высокий уровень тревожности во время тестирования, низкий уровень мотивации или свою некомпетентность при взаимодействии с компьютерным интерфейсом (информационную некомпетентность). Дополнительно результаты такого наблюдения помогают скорректировать процедуру компьютерного тестирования или же подачу стимульного материала, если большинство респондентов выказывают затруднения при ее прохождении. Всё это можно организовать на этапе создания компьютерной версии традиционного теста, чтобы исключить влияние некоторых факторов на результаты эквивалентности двух его форм.

Обращаясь к проблеме психометрических свойств компьютерной версии традиционной методики, К. В. Сугоняев с соавт. предлагает конкретную процедуру статистического анализа, позволяющего, по их мнению, судить об эквивалентности двух форм теста. Исследователями высказывается суждение о том, что сравнение «мер центральной тенденции, вариативности и одномоментной надежности интегральных тестовых оценок» (Сугоняев и др., 2018, с. 8) является недостаточным для проверки сопоставимости результатов тестирования. В качестве метода, призванного доказать эквивалентность двух форм методик, предлагается анализ выполнения теста на пунктовом уровне - сходство/различие «распределения коэффициентов трудности и дискриминативности тестовых заданий, а также паттернов факторных нагрузок пунктов на генеральный фактор» (Сугоняев и др., 2018, с. 18). Таким образом, авторами предпринята попытка обоснования и доказательства необходимого минимума статистических методов для вынесения суждения об эквивалентности компьютеризированной версии методики и ее бумажного аналога. В остальных статьях (табл. 1), как правило, исследователями не поясняется выбор методов математико-статистического анализа (а также дизайн исследования (межгрупповой/внутригрупповой)) с точки зрения достаточности для проверки эквивалентности. И всё же, поскольку условия заполнения компьютерной формы теста отличаются от таковых при заполнении бумажной, и с учетом всех перечисленных выше факторов, способных оказать влияние на процесс выполнения и результат компьютеризированного теста, мы предлагаем не отказываться от идеи проверки валидности и надежности компьютерной версии традиционной методики.

Проведенный анализ результатов исследований, посвященных эквивалентности компьютерной и бумажной форм тестов, также позволяет сделать вывод о том, что большинство из них опубликованы зарубежными специалистами. В отечественной практике, несмотря на обилие программ для компьютерной психодиагностики (Мельничук и Сергеев, н. д.; Будко, Мишин и Трегубова, 2007), данные о процедуре проверки психометрических свойств компьютерных аналогов традиционных тестов приводятся достаточно редко. Также представлено небольшое количество статей, позволяющих оценить качество проверки эквивалентности компьютерных версий методик их бумажным аналогам.

\section{Заключение}

Вне всякого сомнения, психометрические показатели компьютерных версий традиционных методик должны подвергаться проверке. При этом необходимы четкие требования 
ГНЕДЫХ Д. С.

ПРОБЛЕМА ОЦЕНКИ ЭКВИВАЛЕНТНОСТИ КОМПЬЮТЕРНЫХ И ТРАДИЦИОННЫХ ВЕРСИЙ...

РосСИЙСКИй пСИХологИЧЕСКИй ЖУРнАл, 2020, Т. 17, № 1, 44-59. doi: 10.21702/rpj.2020.1.4

к процедуре такой проверки и обоснованные инструкции для специалистов, разрабатывающих компьютерные версии традиционных методик.

Очевидно, что кратких рекомендаций, представленных в Guidelines for computer-based tests and interpretations (APA, 1986), или подробных, но лишенных конкретности инструкций из Standards for educational and psychological testing (American Educational Research Association, American Psychological Association, \& National Council on Measurement in Education, 1999), недостаточно.

На наш взгляд, данные рекомендации должны содержать четкие и обоснованные инструкции по каждому из следующих разделов:

1. Требования к дизайну исследования, необходимые для тщательной проверки эквивалентности бумажной и компьютерной версий психодиагностической методики (количество групп, минимальный размер выборки, последовательность проведения разных форм теста, временной интервал между повторными измерениями, способы проверки конструктной валидности и т. д.).

2. Перечень необходимых и достаточных методов математико-статистической обработки данных, позволяющих констатировать эквивалентность бумажной и компьютерной форм методики.

3. Описание факторов, которые могут повлиять на результаты компьютерного и бумажного тестирования, и рекомендации для организации определенных условий в процессе исследования эквивалентности. В качестве методов, обеспечивающих учет данных факторов, выступают карты наблюдения за поведением респондентов во время выполнения теста, а также стандартизированные анкеты, позволяющие выявить мотивацию, установки по отношению к компьютерной диагностике, уровень компьютерной грамотности и т. п. (например, опросник на выявление мотивации к тестированию (Testing Motivation Questionnaire) (Chua, 2012), опросник «Знакомство с компьютером» (Computer Familiarity Questionnaire) (Mazzeo et al., 1992)). Это поможет исключить влияние дополнительных переменных на результаты тестирования и получить более достоверные данные об эквивалентности традиционных и компьютерных форм психодиагностических тестов.

\section{Литература}

Анастази, А. и Урбина, С. (2009). Психологическое тестирование. Санкт-Петербург: Питер.

Бабанин, Л. Н. (2010). Проблема эквивалентности традиционных и компьютеризированных психологических методик. Экспериментальная психология в России: традиции и перспективы. Доступ 27 октября 2019, источник http://psyjournals.ru/exp collection/issue/32974.shtml

Батурин, Н. А. и Мельникова, Н. Н. (2009). Технология разработки тестов. Часть І. Вестник ЮУрГУ. Серия Психология, 6(30), 4-14.

Батурин, Н. А. и Мельникова, Н. Н. (2009). Технология разработки тестов. Часть ІІ. Вестник ЮУрГУ. Серия Психология, 7(42), 11-25.

Батурин, Н. А. и Мельникова, Н. Н. (2010). Технология разработки тестов. Часть III. Вестник ЮУрГУ. Серия Психология, 8(4), 4-18.

Батурин, Н. А. и Мельникова, Н. Н. (2010). Технология разработки тестов. Часть IV. Вестник ЮурГу. Серия Психология, 11(40), 13-28.

Батурин, Н. А. и Мельникова, Н. Н. (2011a). Технология разработки тестов. Часть V. Вестник ЮурГУ. Серия Психология, 12(5), 4-14. 
ГНЕДЫХ Д. С.

ПРОБЛЕМА ОЦЕНКИ ЭКВИВАЛЕНТНОСТИ КОМПЬЮТЕРНЫХ И ТРАДИЦИОННЫХ ВЕРСИЙ...

Российский психологИчЕский жУРнАл, 2020, Т. 17, № 1, 44-59. doi: 10.21702/rpj.2020.1.4

ОБЩАЯ ПСИХОЛОГИЯ, ПСИхОЛОГИЯ ЛИЧНОСТИ, ИСТОРИЯ ПСИХОЛОГИИ

Батурин, Н. А. и Мельникова, Н. Н. (20116). Технология разработки тестов. Часть VI. Вестник ЮУрГУ. Серия Психология, 13(18), 48-59.

Будко, В. Н., Мишин, Д. Ю. и Трегубова, Т. А. (2007). Тестирующий программный комплекс готовности студентов и школьников к обучению в вузе. Инфокоммуникационные технологии, 5(3), 167-169.

Вассерман, Л. И., Иовлев, Б. В. и Червинская, К. Р. (2010). Компьютерная психодиагностика в теории и практике медицинской психологии: этапы и перспективы развития. Сибирский психологический журнал, 35, 20-24.

Ермаков, С. С. (2016). Особенности компьютерной диагностики интеллектуальных способностей учащихся VI-IX классов. Психологическая наука и образование, 8(4), 199-207. doi: 10.17759/psyedu.2016080419

Иовлев, Б. В., Новожилова, М. Ю., Червинская, К. Р. и Щелкова, О. Ю. (2006). Методологические аспекты изучения эффективности компьютерной психодиагностики. Вестник СанктПетербургского университета. Сер. 6. Философия, политология, социология, психология, право, международные отношения, 2, 115-124.

Кибальченко, И. А., Устинов, Д. А. и Шаповалов, С. Н. (2004). Компьютерная психодиагностика как условие развития одаренности. Известия ТРТУ, 6, 301-303.

Кононова, В. Н. и Нахаева, И. В. (2013). Исследование психометрической эквивалентности двух версий проективной психодиагностической методики - традиционной («цветовое зеркало») и компьютеризированной («цветомер личности»). Вестник Московского университета. Серия 14. Психология, 1, 136-151.

Мельничук, А. и Сергеев, В. (н. д.). Современные компьютерные системы для эффективной оценки персонала. Психология и бизнес. Доступ 13 декабря 2019, источник https://psycho.ru/library/93

Митина, О. В. и Сорокина, В. В. (2015). Ценности старшеклассников: разработка компьютерного диагностического инструментария. Вестник Московского университета. Серия 14. Психология, 1, 42-59.

Ромек, В. Г. и Сатин, Д. К. (2000). Сохранение надежности многофакторных тестов при их использовании в сети Интернет. Психологический Журнал, 21(2), 70-75.

Сугоняев, К. В., Радченко, Ю. И. и Соколов, А. А. (2018). Добровольное Интернет-тестирование как источник валидных оценок группового психометрического интеллекта. Сибирский психологический журнал, 69, 6-32. doi: 10.17223/17267080/69/1

American Educational Research Association, American Psychological Association, \& National Council on Measurement in Education. (1999). Standards for educational and psychological testing. Washington, DC: American Educational Research Association.

Andersson, G., Kaldo-Sandström, V., Ström, L., \& Strömgren, T. (2003). Internet administration of the Hospital Anxiety and Depression Scale in a sample of tinnitus patients. Journal of Psychosomatic Research, 55(3), 259-262. doi: 10.1016/S0022-3999(02)00575-5

APA (1986). Guidelines for computer-based tests and interpretations. Washington, DC: Author.

Bartram, D. (1994). Computer-based assessment. In C. L. Cooper (Ed.), International review of industrial and organizational psychology (pp. 31-69). London: Wiley.

Campbell, D. T., \& Stanley, J. C. (1963). Experimental and quasi-experimental designs for research. Chicago: Rand McNally \& Company.

Chua, Y. P. (2012). Effects of computer-based testing on test performance and testing motivation. Computers in Human Behavior, 28(5), 1580-1586. doi: 10.1016/j.chb.2012.03.020 
Ford, B. D., Vitelli, R., \& Stuckless, N. (1996). The effects of computer versus paper-and-pencil administration on measures of anger and revenge with an inmate population. Computers in Human Behavior, 12(1), 159-166. doi: 10.1016/0747-5632(95)00026-7

George, C. E., Lankford, J. S., \& Wilson, S. E. (1992). The effects of computerized versus paper-andpencil administration on measures of negative affect. Computers in Human Behavior, 8(2-3), 203-209. doi: 10.1016/0747-5632(92)90004-X

Hays, S., \& Mccallum, R. S. (2005). A comparison of the pencil-and-paper and computer-administered Minnesota Multiphasic Personality Inventory-Adolescent. Psychology in the Schools, 42(6), 605-613. doi: 10.1002/pits.20106

Honaker, L. M. (1988). The equivalency of computerized and conventional MMPI administration: A critical review. Clinical Psychology Review, 8(6), 561-577. doi: 10.1016/0272-7358(88)90081-5

Květon, P., Jelínek, M., Vobořil, D., \& Klimusová, H. (2007). Computer-based tests: The impact of test design and problem of equivalency. Computers in Human Behavior, 23(1), 32-51.

Mazzeo, J., Druesne, B., Raffeld, P. C., Checketts, K. T., \& Muhlstein, A. (1992). Compatibility of computer and paper-and-pencil scores for two CLEP ${ }^{\circledR}$ general examinations. ETS Research Report Series, 1. doi: 10.1002/j.2333-8504.1992.tb01446.x

Meade, A. W., Michels, L. C., \& Lautenschlager, G. J. (2004, April). Are online and paper-and-pencil personality tests truly comparable? Symposium presented at the 19th Annual Conference of the Society for Industrial and Organizational Psychology. Chicago, IL.

Russell, M., Goldberg, A., \& O'connor, K. (2003). Computer-based Testing and Validity: A look back and into the future. Assessment in Education: Principles, Policy \& Practice, 10(3), 279-293. doi: 10.1080/0969594032000148145

Solomon, R. L. (1949). An extension of control group design. Psychological Bulletin, 46(2), 137-150. doi: $10.1037 /$ h0062958

The International Testing Commission. (2006). International guidelines on computer-based and Internet-delivered testing. International Journal of Testing, 6(2), 143-171. doi: 10.1207/ s15327574ijt0602 4

van de Looij-Jansen, P. M., Goldschmeding, J. E. J., \& Jan de Wilde, E. (2006). Comparison of anonymous versus confidential survey procedures: Effects on health indicators in Dutch adolescents. Journal of Youth and Adolescence, 35, 652-658. doi: 10.1007/s10964-005-9027-0

Vecchione, M., Alessandri, G., \& Barbaranelli, C. (2012). Paper-and-pencil and web-based testing: The measurement invariance of the Big Five Personality Tests in applied settings. Assessment, 19(2), 243-246. doi: 10.1177/1073191111419091

Žitný, P., Halama, P., Jelínek, M., \& Květon, P. (2012). Validity of cognitive ability tests - comparison of computerized adaptive testing with paper and pencil and computer-based forms of administrations. Studia Psychologica, 54(3), 181-194.

Конфликт интересов отсутствует 